Pippa Powell ${ }^{1}$, Ravijyot Saggu², Steve Jones ${ }^{1,3}$, Marco Clari $^{4}$, Isabel Saraiva ${ }^{5}$, Georgia Hardavella ${ }^{6}$, Kjeld Hansen ${ }^{1,7}$, Hilary Pinnock ${ }^{8}$

kjeld.hansen@europeanlung.org

@EuropeanLung

\title{
Viewpoint
}

\section{Discussing treatment burden}

Treatment burden is defined as "the workload of healthcare and its effect on patient functioning and well-being" [1, 2]. It has also been defined as "the self-care practices that patients with chronic illness must perform to respond to the requirements of their healthcare providers, as well as the impact that these practices have on patient functioning and well-being" [3]. In this issue of Breathe, there are several articles addressing treatment burden from different perspectives. This Viewpoint article focusses on the discussions between the patient and their healthcare professional (HCP). What are the important topics to cover in discussions about treatment burden? What are the barriers to these discussions? What facilitates a productive conversation about treatment burden? The authors of this article include three patients (with asthma, COPD and idiopathic pulmonary fibrosis (IPF), four HCPs representing different specialties (primary care, secondary and tertiary care, nursing and pharmacy) and a representative of the European Lung Foundation (ELF). This article is based on a video discussion between the authors; it was then refined via e-mail by all the authors.

\section{What key factors about treatment burden should be discussed during a consultation when medications are being prescribed?}

\section{Disease trajectory related to medicines}

From the outset of any discussion regarding medication, it is important to relate it to the patient's diagnosed condition and to avoid medical jargon. It may not be possible to cure the condition, but the HCPs should explain the realistic aims of treatment, manage the patient's expectations and suggest strategies to enable the patient to manage their condition and improve their quality of life. Crucially, the patient needs to be told if this requires using medicines regularly and at specific hours of the day and/or making changes to lifestyle and work-related activities. It is equally important to highlight the implications if no changes are made (e.g. if the patient continues to smoke). Patients may need to be aware that some treatments are life-long, and that stopping or modifying treatment could result in worsening of their condition.

\section{Potential benefits of treatment}

It is helpful to discuss broadly what treatments are available to the patient (both pharmacological and non-pharmacological) with the aim of personalising the approach to the individual. Understanding the patient's perception of the severity of their condition, their expectations from treatment and the magnitude of potential benefits is important. For example, some patients may not take medication as intended (or at all), believing that their condition is not
Cite as: Powell P, Saggu R, Jones $S$, et al. Discussing treatment burden. Breathe 2021; 17: 200284.

@ERSpublications

Healthcare professionals should help patients to reach informed decisions about treatments in

order to maximise benefits while minimising treatment burden https://bit.ly/2XRtRPK

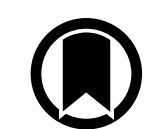

CrossMark 
serious or that they have little to gain. This may be particularly difficult when the benefits (e.g. having no immediate change in symptoms with preventative treatment, such as in asthma) are not immediately apparent but adherence to early treatment results in better long-term outcomes. Burden is relevant and may be a trade-off: for example, a patient may be willing to take several pills a day if their condition is serious, but not if they consider their condition to be mild. HCPs should communicate the potential treatment benefits and relevant treatment timeframe in a way that the patient can understand, accounting for any learning (or other) disability/language barriers, in order to ensure that the patient has a clear understanding and can work closely with their HCP to commit to the treatment targets.

\section{Potential side effects and impact on quality of life}

Any medication prescribed should be the "best fit" for the patient. To reach this decision, the patient must understand the medication's potential side effects so they can weigh up the possible impact on their quality of life. A good example of this is in IPF, for which the two available antifibrotic drugs have very different side effects and the choice may depend on the patient's assessment of the likely impact of side effects. It is vital that patients know who to contact to discuss concerns (and how to do so) and for practical advice on how to manage side effects. Similarly, regular monitoring, especially in the early stages ensures that side effects are recognised promptly and managed efficiently. In patients who, themselves, have barriers to understanding (young children or patients with severe cognitive impairment), it is important to have this conversation with their carer(s) whilst involving the patient to the extent that understanding allows.

\section{The possibility to stop ineffective treatments}

A treatment "trial" can be carried out with agreement to stop the medication if clinical goals are not achieved and/or benefit is not felt by the patient within a specific timeframe. Realistic treatment goals of a new medication must be clearly defined for both the patient and the HCP, recognising that the patient's perception of benefit may differ from a clinical definition of effectiveness. A patient, for example, may want to improve dayto-day function or to prioritise extended life, while the HCP may judge effectiveness as a change in physiological parameters. More generally, all medicines should be reviewed regularly (annually as a minimum) to check that they are still appropriate and to ensure their usage remains meaningful for the patient.

\section{Practicalities}

The HCP should explore the patient's perceptions and barriers to adopting interventions (both medicine and any other recommendations). Does it fit with their daily routines? Can the dosage regimen be simplified to avoid a complex schedule with many and/or frequent medicines? It is important that the HCP and patient work in partnership to dispel myths and agree a way forward that fits with both the patient's medical needs and their lifestyle and working routine.

The method of administration (e.g. tablet, injection, inhaler) may be important to the patient. It needs to be practical, convenient and acceptable, and as consistent as possible. For example, if an inhaler device is changed the patient has to be re-educated on how to take the medication to minimise the risk of sub-optimal technique.

\section{Access to medication}

Some medications are only available from a specialist. Arrangements for repeat prescribing can create additional burdens on the patient, especially if the specialist care centre is far from home or specific monitoring of the patient is required. This has been especially relevant during the coronavirus disease 2019 (COVID-19) pandemic due to limitations to accessing healthcare facilities. Close collaboration between primary and specialist care and e-health technologies can be used to minimise patient burden in requesting and receiving repeat prescriptions.

Although cost may not be an issue that is discussed during a consultation, the HCP should be aware whether their patient will have to pay for prescriptions (and this may vary from condition to condition and country to country), as this can be a significant barrier for some patients. Financial constraints preventing the patient's access to medication may be addressed with the help of social services or relevant services depending on the country.

\section{Polypharmacy, multimorbidities and medication interactions}

Polypharmacy is the situation of having many medications (or many different forms of medicine, e.g. inhalers, tablets, patches and liquids), which adds to the treatment burden for a patient. Polypharmacy is sometimes necessary but, if additional medication is recommended, the reasons should be clearly explained to the patients and, if appropriate, another medication should be stopped. Tools such as "STOPPSTART" (Screening Tool of Older Persons' Prescriptions and Screening Tool to Alert to Right Treatment) may be considered as a guide to de-prescribing [4, 5]. These tools offer criteria to help HCPs to review potentially inappropriate medications in the elderly and have been endorsed as best practice by some organisations. 
Many patients have multimorbidity, and HCPs should always discuss potential interactions of the medication being prescribed with other treatments. This may require colleagues working in primary care to mediate between multiple specialists. Patients can be very concerned about possible interactions between the different medications that they are taking. Just explaining that computer prescribing software generates warnings and that this will be flagged up and considered by the HCP can be reassuring. In countries where computer software is not available, HCPs need to ensure a different safety net is in place to avoid interaction risks. Interactions between patient factors (e.g. renal/liver function, disability, attitude to risk, experience of illness, values/beliefs and social circumstances) and medicine can be avoided by a pharmacist's check to reduce and mitigate unnecessary risk to patients.

\section{Non-pharmacological interventions}

Non-pharmacological interventions, such as pulmonary rehabilitation, breathing retraining or social prescribing (e.g. attending a gym), may help reduce pharmacological treatment burden for a patient. These should always be considered as adjuncts to conventional therapy where possible. On some occasions, patients wish to discuss nonpharmacological interventions deriving from their cultural backgrounds or internet searches. HCPs need to gather information from the patient and be cautious about considering these options as well as ensuring they are safe and suitable for the patient.

\section{Signposting to reputable resources of information}

Patients can now access information in many different ways about their condition and their treatment. To ensure that they use trustworthy and reliable information, the HCP should signpost patients to organisations and websites where they can find reliable evidence-based information.

\section{What are the barriers to discussions about treatment burden?}

One of the major barriers for both HCPs and patients is the (limited) time available during consultations, which makes detailed discussions on treatment burden difficult. HCPs should be prepared to recommend further sources of reliable information. For example, support groups (where they exist) can provide a supportive environment in which to share experiences (e.g. of side effects) with other patients and allow extended time for discussion and feedback.
The official "patient information leaflets" (PILs) that accompany medication ought to be useful sources of information, but patients are often put off by the long list of adverse side effects and by a lack of balanced information about risks and benefits. A particular issue which causes anxiety and concern for patients is that the PIL may not give information on unlicensed uses of the medicine. HCPs should pre-empt this, explain any discrepancies and not rely on these leaflets as the sole source of information for patients on their medication. Some patient organisations publish leaflets on key medications with input from HCPs.

\section{For patients}

Patients may lack the knowledge, understanding, skills and confidence to use health information effectively. HCPs need to take this into account by providing information in a form and format patients can understand, preferably in their native language. They should also address specific barriers, such as learning disabilities and reduced physical/ functional/cognitive capacity, which mean patients may struggle to comprehend the information being provided. Conversely, there can be challenges associated with "expert patients" who have set ideas and specific (sometimes unrealistic) expectations, which prohibit an open conversation with the HCP.

Patients may fear being blamed by the HCP; for example, for being a smoker or overweight. They may feel guilt or shame for not taking the medication that has been prescribed to them. A non-judgmental relationship and rapport between the HCP and patient is vital, allowing patients to explain about the treatment burden, the difficulties they have with regular medication or the reason that they have chosen to discontinue medication. $\mathrm{HCPs}$ should be careful with the language used in these discussions. Other factors that might act as a barrier for patients to discuss treatment burden include lack of self-efficacy, demotivation and lack of confidence.

One final point highlighted by patients, is that it can be really difficult to listen to details about medication when trying to take in and process the enormity of a diagnosis of a life-limiting disease such as IPF (which has a 3-5 year prognosis) or cancer. Patients who are worried about their disease are often unable to listen well and may not fully absorb the information provided or think to raise issues of concern. Taking a friend or family member into a consultation can help to ensure that the patient has someone supporting them, listening to information discussed and prompting the patient to ask questions or explain their worries.

\section{For HCPs}

Offering choice can be difficult for HCPs who are often limited in what they can prescribe by the need 
to follow guidelines or organisational constraints related to cost or medication availability in their settings.

HCPs may lack training in communication skills or would benefit from learning techniques such as motivational interviewing. Such communication skills should be a fundamental component of all medical, nursing or other HCP training and continued medical education (CME).

There is often a lack of integration in healthcare systems, which is exacerbated by poor IT infrastructure. This can result in different specialists not having complete information about the patient when reviewing them. In some countries, primary and specialist care are not linked through IT systems and information cannot be shared in real time. It is essential that all HCPs treating a patient are informed about other aspects of their care. This is particularly true with patients who have other chronic conditions.

\section{How to have a productive discussion about treatment burden}

There have been many points highlighted here that can help HCPs and patients to discuss the issue of treatment burden. There have also been a number of publications on the topic [6, 7]. Table 1 outlines the top tips from the authors of this paper to facilitate productive conversation.

\section{Conclusion}

There are many aspects of treatment burden that should be considered by HCPs when prescribing new medications to patients. These include having a common understanding of the goals of treatment, the risk of adverse side effects and potential impact

Table 1 Tops tips for discussion between the healthcare professional and the patient about treatment burden

\section{For the patient}

Prepare questions and practice explaining about concerns before your consultation

Take a carer or friend with you to consultations. Brief them about your questions and concerns and ask them to help listen to the information provided

Think about treatment goals and be clear what is important to you (e.g. being less breathless, being able to run)

Ask for the names of other HCPs (e.g. specialist nurse) who you can discuss this with in more detail in the future

Ensure that you understand why a treatment is being recommended and ask for further information to help you make decisions about this if required

Read and learn from trusted/reputable resources. Ask about good sources of information

Ask about side-effects, and about how likely they are to happen. Check what you should do if you are experiencing a side-effect

Explain any factors that you feel may make it harder for you to take your medicines or follow the treatment recommendations given

Ask if you think that one of your medications is no longer needed or is causing side-effects

Ask about written information (such as action plans) that could help you follow advice

Medication reviews are important for safe use of medications - use them to discuss any concerns that you have about your treatment

\section{For the healthcare professionals}

Patients with chronic conditions have to adjust to the implications of the diagnosis on their life and lifestyle. Be sensitive with how you communicate this

Encourage the patient to bring a companion; and encourage them to write down key information

Discuss lifestyle behaviours of the patient: what are their priorities? This will help to guide the best medication, taking into account issues such as side-effects, monitoring requirements, cost, impact on driving and family

Provide details, where possible, for a nurse or pharmacist who will have the time to have a longer/further discussion with the patient; but ensure the messages are consistent

Explain clearly what treatments are available so patients can make an informed choice. Remember that limited health literacy is common even without specific challenges or learning disabilities

Provide the patient with details of a trusted patient organisation or peersupport group from which they can gain input and further advice

Do not wait to be asked about side-effects. Tell patients about anything common or potentially serious and ensure they know what to do if a problem develops

Explore non-adherence in a non-judgemental way to agree with the patient how to gain benefit from treatment, personalised to the individual's circumstances

Agree with the patient if specific medications can be stopped or regime modified (using tools such as STOPP/START). Regularly review treatments to de-prescribe, if appropriate

Provide simple, written follow-up advice, including self-management plans (e.g. asthma action plans [8]), which can empower patients to be partners in their care

Using a qualitative measure of treatment burden at annual reviews could offer an opportunity to raise issues that are bothering the patient [9] 
on lifestyle. Healthcare professionals should help patients to reach informed decisions about treatments in order to maximise benefits while minimising the treatment burden.

\title{
Key points
}

- There are many aspects of treatment burden that should be considered by healthcare professionals (HCPs) when prescribing new medications to patients. These include having a common understanding of the (realistic) goals of treatment, the risk of side-effects and the potential impact on lifestyle.

- Major barriers to the discussion of treatment burden include lack of time, limited understanding on the part of the patient, and limited communication skills and understanding of the patient's perspective by the HCP.

- Patients and HCPs should prepare for a discussion about new medications to ensure that the burden of treatment can be minimised.

\begin{abstract}
Affiliations
Pippa Powell ${ }^{1}$, Ravijyot Saggu ${ }^{2}$, Steve Jones ${ }^{1,3,4}$, Marco Clari $^{5}$, Isabel Saraiva ${ }^{6}$, Georgia Hardavella ${ }^{7}$, Kjeld Hansen ${ }^{1,8}$, Hilary Pinnock ${ }^{9}$

${ }^{1}$ European Lung Foundation, Sheffield, UK. ${ }^{2}$ University College London Hospitals NHS Foundation Trust, London UK. ${ }^{3}$ EU IPFF, Brussels, Belgium. ${ }^{4}$ Action for Pulmonary Fibrosis (APF), Peterborough, UK. ${ }^{5}$ Dept of Public Health and Pediatrics, University of Torino, Turin, Italy. ${ }^{6}$ Associaçă Portuguesa de Pessoas com DPOC e outras Doenças Respiratórias Crónicas (RESPIRA), Lisbon, Portugal. ${ }^{79}$ th Dept of Respiratory Medicine, Athens Chest Diseases Hospital Sotiria, Athens, Greece. ${ }^{8}$ Dept of Technology, Kristiania University College, Oslo, Norway. ${ }^{9}$ Usher Institute, University of Edinburgh, Edinburgh, UK.
\end{abstract}

\section{Conflict of interest}

P. Powell is an employee of the European Lung Foundation. R. Saggu has nothing to disclose. S. Jones has nothing to disclose. M. Clari has nothing to disclose. I. Saraiva has nothing to disclose. G. Hardavella has nothing to disclose. K. Hansen is the Chair of the European Lung Foundatione. H. Pinnock has nothing to disclose.

\section{References}

1. Eton DT, de Oliveira DR, Egginton JS, et al. Building a measurement framework of burden of treatment in complex patients with chronic conditions: a qualitative study. Patient Relat Outcome Meas 2012; 3: 39-49.

2. Dobler CC, Nathan H, Maguire CA, et al. Treatment burden should be included in clinical practice guidelines. BMJ 2018; 363: k4065.

3. Gallacher K, Morrison D, Jani B, et al. Uncovering treatment burden as a key concept for stroke care: a systematic review of qualitative research. PLoS Med 2013; 10: e1001473.

4. Ryan C. The basics of the STOPP/START criteria. www.pcne. org/upload/ms2011d/Presentations/Ryan\%20pres.pdf Date last accessed: 24 November 2020.

5. O'Mahony D, O'Sullivan D, Byrne S, et al. STOPP/START criteria for potentially inappropriate prescribing in older people: version 2. Age Ageing 2015; 44: 213-218.
6. Working together to help patients make the most of medicines. Report of the Royal College of Nursing and Royal Pharmaceutical Society Joint Summit. 2014. www.rpharms. com/Portals/0/RPS\%20document\%20library/Open\%20 access/Hub/rps-make-the-most-of-medicines-web.pdf Date last accessed: 24 November 2020.

7. Liberating the NHS: no decision about me without me. https:// assets.publishing.service.gov.uk/government/uploads/ system/uploads/attachment_data/file/216980/Liberatingthe-NHS-No-decision-about-me-without-me-Governmentresponse.pdf Date last accessed: 24 November 2020.

8. Gruffydd-Jones K, Hansen K. Working for better asthma control: how can we improve the dialogue between patients and healthcare professionals? Adv Ther 2020; 37: 1-9.

9. Sheehan OC, Leff B, Ritchie CS, et al. A systematic literature review of the assessment of treatment burden experienced by patients and their caregivers. BMC Geriatr 2019; 19: 262. 